\title{
De los dioses a los hombres: Un recorrido histórico del descubrimiento de los elementos químicos
}

\section{From Gods to men: A historical overview of the discovery of the chemical elements}

\author{
I. Pellón González \\ Universidad del País Vasco (UPV/EHU). Departamento de Ingeniería Química y del Medio Ambiente. ETS de Náutica y \\ Máquinas Navales, C/ Mª Díaz de Haro, n 68, 48920 - Portugalete. Email: ines.pellon@ehu.es
}

\section{RESUMEN}

Llegar hasta la aparentemente sencilla Tabla Periódica de los Elementos le ha costado a la humanidad enormes esfuerzos a lo largo de miles de años. En este trabajo se recorre la historia del descubrimiento de los elementos químicos desde la Prehistoria hasta nuestros días, reflejando las controversias que se suscitaron y reivindicando el importante trabajo que realizaron los alquimistas en el progreso de los conocimientos, ya que la alquimia tuvo un período de existencia de muchos miles de años, mientras que la "Química" oficialmente establecida como ciencia en el siglo XVIII consta de sólo unos cientos de años. Aún así, al realizar un balance de la progresión del descubrimiento y aislamiento de los elementos químicos a lo largo de la Historia se puede comprobar el elevado número de elementos identificados en los siglos XIX y XX, reflejo del perfeccionamiento de las técnicas instrumentales que facilitaron esta labor.

Palabras clave: Historia de la Química; Elementos Químicos

\begin{abstract}
To achieve the apparently simple Periodic Table of the Elements has implied tremendous efforts over thousands of years. In this paper we present a brief history of the discovery of the chemical elements from prehistory to the present day, revealing the controversies that arose on the way and claiming the important work performed by alchemists in the advancement of knowledge. This is especially important if we consider that alchemy had a period of existence of many thousands of years, while the "Chemistry", officially established as a science in the eighteenth century, has operated as such for only a few hundred years. Even so, if we consider the progress of discovery and isolation of chemical elements throughout history, it can be observed that the number of elements identified is achieved mainly in the nineteenth and twentieth centuries, reflecting the development of instrumental techniques, that facilitated this task.
\end{abstract}

Keywords: History of Chemistry; Chemical Elements

Citation / Cómo citar este artículo: I. Pellón González (2014). De los dioses a los hombres: Un recorrido histórico del descubrimiento de los elementos químicos. Estudios Geológicos 70(2): e015. http://dx.doi.org/10.3989/egeol.41718.316.

Copyright: (c) 2014 CSIC. This is an open-access article distributed under the terms of the Creative Commons Attribution-Non Commercial (by-nc) Spain 3.0 License. 


\section{A modo de Introducción: Un viaje desde la Prehistoria hasta la Edad Media}

Los primeros materiales empleados por los seres humanos fueron la madera, la piedra y los huesos tallados y trabajados, así como el hierro meteórico, el oro y el cobre nativos, que se moldeaban golpeándolos en frío. Pero llegó un momento en el que para poder utilizar los metales puros era necesario alterar profundamente su estado natural, hecho que supuso la aparición de la metalurgia, punto de inflexión en la historia de la humanidad. Debido a su maleabilidad, el cobre nativo resulta muy fácil de manipular y probablemente por ello fue el primer metal que se empleó. Cuando su uso se generalizó nació la denominada Edad de Cobre o Calcolítico (ca. 8000 a.C.4000 a.C.), en la que convivieron durante mucho tiempo la cultura lítica con los utensilios de este metal. La presencia casual de arsénico en algunas de las menas de cobre utilizadas llevó al descubrimiento de cómo formar aleaciones con él y con otros elementos como el antimonio, el plomo o el estaño, metal con el que se obtiene el bronce. En un principio este nuevo material sólo se utilizó para fabricar objetos decorativos, pero al quedar patente que su dureza era mucho mayor que la del cobre y la de la piedra, su uso se generalizó. En la denominada Edad de Bronce ( $c a .4000$ a.C.-700 a.C.), la demanda de esta aleación originó la denominada "ruta de los metales", que unía el Mediterráneo con el centro y el norte de Europa y llegaba hasta la Península Ibérica en busca de los preciados cobre y estaño. Este recorrido servía para comercializar tanto los lingotes como los productos ya terminados y se unía a la que generaba el comercio del apreciado ámbar, cuyos yacimientos se encontraban en el norte de Europa (Fernández Castro, 1997).

Cuando se produjeron dificultades en el abastecimiento de cobre y estaño para fabricar bronce se sustituyó por el hierro, metal ya conocido pero que presentaba más dificultades que el cobre para su transformación a partir de sus minerales por su elevado punto de fusión $\left(1538^{\circ} \mathrm{C}\right)$. Hay pruebas arqueológicas que demuestran que el hierro proveniente de los meteoritos se empleó en Egipto en el año 4000 a.C. así como en la cultura mesopotámica, donde consideraban que este metal era un "Regalo de los Dioses" porque provenía de cielo. El descubrimiento de la fundición condujo a la predominancia del uso de hierro para fabricar herramientas y armas, lo que dio lugar al inicio de la Edad de Hierro, etapa que duró hasta la expansión del imperio romano. A lo largo de los siglos se conoció cómo obtener otros elementos, de tal forma que al llegar al Renacimiento se utilizaban doce: cobre, hierro, oro, plata, estaño, arsénico, plomo, azufre, mercurio, zinc, antimonio y carbono, que se empleaba en forma de carbón vegetal para la reducción del cobre, zinc y estaño.

Gracias a la utilidad de los productos que fabricaban, los artesanos del metal eran muy respetados en la antigua cultura mesopotámica, donde también se conocía la manera de obtener perfumes, medicinas, detergentes o pigmentos para teñir tejidos. Asimismo eran capaces de preparar vidrios coloreados y gemas artificiales, de obtener una imitación de plata por blanqueo del cobre y de realizar fermentaciones. Es en esta civilización donde se tiene noticia por primera vez de un "Arte Sagrado" que, según la tradición, fue confiado a los humanos por un ángel caído del cielo que quedó cautivado por la hermosura de las mujeres terrenales (Moorey, 1994).

En el Egipto arcaico se explotaron principalmente los yacimientos del norte de Nubia, en cuyos restos hay evidencias de crisoles, yunques, cinceles y diversos objetos metálicos, aunque carecemos de testimonios escritos que reflejen las técnicas empleadas por los metalurgistas y orfebres. Sin embargo, tenemos la inmensa suerte de contar con un testigo de excepción que describe con exactitud los métodos que utilizaban para extraer y fundir los metales: los dibujos de la tumba del visir Rkh-mi-Ra (ca. 1460-1430 a.C), entre los que se representan los talleres de los metalúrgicos con cuatro hornos alimentados con carbón, cuyo fuego es avivado mediante fuelles de madera y cuero manejados con los pies y con cuerdas. Sabemos que se añadía sal y plomo para eliminar los residuos de plata al formarse cloruro argéntico que, junto con el plomo, constituía las escorias. Al cabo de cinco días el crisol se sacaba del fuego y el oro fundido se vertía en pequeñas vasijas en las que se enfriaba y solidificaba (Klemm, 2013).

Por otro lado, los importantes avances que la metalurgia china consiguió en fechas muy tempranas pueden ejemplificarse en el pleno desarrollo de la copelación a comienzos del siglo III a.C. para refinar el oro y la plata mediante su aleación con plomo y 
la oxidación posterior del plomo fundido para separarlo del metal precioso (Needham, 1978).

Asimismo son destacables los conocimientos alquímicos que se desarrollaron en la antigua India (Chakravarti, 2003), saberes que se transmitieron a otras civilizaciones posteriores como la etrusca, griega y romana (Bensinger, 2014), en las que además de desarrollar todos los aspectos prácticos del "Arte Sagrado", se intentó descubrir cuál era la estructura última de la materia. Desde el atomismo griego se llegó a la idea de los cuatro elementos (agua, aire, fuego y tierra) y sus cualidades (frío, húmedo, caliente y seco), con las que se buscaba explicar los diferentes estados de las sustancias. Hoy en día se considera que la alquimia surgió en China y en Egipto de forma independiente, y gracias a esta última civilización se transmitieron los conocimientos alquímicos a la cultura árabe. En la ciudad de Harran, situada en la frontera turco-siria, se compilaron los tratados antiguos sobre medicina, astronomía, filosofía y alquimia a lo largo del siglo séptimo. Zona de confluencia de numerosas culturas, destacó por ser un importante centro metalúrgico donde se trabajaban los metales traídos de Asia Menor, Kurdistán y Persia. Se cree que fueron los árabes quienes acuñaron el término Al-kimiya o Arte sagrado, que los traductores latinos transformaron en alkimia, alquimia, alchimia o alchemia. En árabe, el prefijo $\mathrm{Al}$ es el artículo determinado para el sustantivo griego chêmeia, que era la palabra empleada para designar a la fundición de metales (Newman \& Principe, 1998).

Hubo varios alquimistas árabes importantes, siendo el más conocido el iraní Abu Musa Jabir ibn Hayyan (ca. 721-ca. 815), denominado Geber por los latinos. Geber escribió numerosos tratados alquímicos en los que detalló varias operaciones de química práctica, como el método de preparación del aqua fortis (ácido nítrico) para separar el oro de la plata, los diferentes usos de distintas sustancias como los álcalis (hidróxidos), el vitriolo (ácido sulfúrico) o el vitriolo verde (sulfato de cobre), la descripción de los colores que produce el cobre en la llama, la fabricación de acero, la purificación de metales, la preparación de barnices, tintes o perfumes, las técnicas de destilación, o el empleo del dióxido de manganeso para fabricar vidrio incoloro entre otros (Al-Khalili, 2012).

\section{El "Arte Sagrado" desde la Edad Media hasta el siglo XVI}

Los alquimistas medievales europeos llegaron a perfeccionar numerosas técnicas de laboratorio, desarrollaron los procesos químicos básicos y describieron numerosos aparatos para diferentes operaciones químicas. A pesar de que estas técnicas se conocían desde hacía siglos, ellos fueron quienes las plasmaron por primera vez en sus manuscritos (Principe, 2013), cuyo estudio en profundidad ha permitido la traducción del lenguaje hermético y se ha podido establecer la conexión entre su idioma y el nuestro. Poco a poco y gracias a la estupenda labor de los equipos multidisciplinares formados por historiadores y químicos, se está empezando a conocer la auténtica dimensión del trabajo experimental de los alquimistas (Martinón-Torres, 2003 y 2008).

Durante los siglos XII, XIII y XIV la Península Ibérica se convirtió en un crisol de civilizaciones en el que se tradujeron numerosos libros que contenían conocimientos de toda índole. Especial importancia tuvo la Escuela de Traductores de Toledo, a la que se encontraba vinculado G. de Cremona (1114-1187), especializado en traducir textos alquímicos como el popular Libro de la composición de la alquimia (1142) (Martín Reyes, 2004). Esta transmisión de conocimientos se produjo gracias al trabajo de los monjes franciscanos y dominicos, quienes realizaban las tareas de recopilación y custodia de los libros en los monasterios. Entre ellos destacaron como alquimistas los franciscanos E. Buonbarone o de Asís (ca. 1180-1253), B. de Iseo (1180-1280) y R. Bacon (1214-1294), autor de la denominada Opus Maius ("Gran Obra"), cuyas 840 páginas tratan sobre ciencias naturales, gramática, lógica, matemáticas, física y filosofía, en las que su sexta parte es una completa revisión sobre la alquimia. Asimismo sobresalieron en el "Arte" los dominicos V. de Beauvais (1190-1264) y Alberto Magno (ca. 11931280), quien delimitó claramente los ámbitos de la fe y de la razón y fue un reconocido investigador. Denominado Doctor universalis, en sus textos refleja la producción de metales, el estudio de los principios del azufre y del mercurio, la descripción de numerosas operaciones químicas y el estudio del arsénico, la sal amoniacal (amoníaco), los álcalis, el cinabrio, etc. Además, Alberto Magno fue el primer 
europeo en reflejar por escrito cómo aislar el arsénico en 1250 (Heines, 1958).

Desgraciadamente para los alquimistas, la propagación de la herejía joaquinista entre las órdenes religiosas llevó a la primera prohibición oficial de las artes alquímicas en 1272 , que fue continuada por varias acciones para impedir las falsificaciones y la charlatanería. Por ejemplo, el alquimista J. Duèze (1244-1334), que fue nombrado Papa (Juan XXII) en 1316, promulgó el decreto pontificio Spondent quas non exhibent (1317), que específicamente prohibía la fabricación de oro falso.

Los siglos continuaron pasando y el médico, naturalista y alquimista Th. B. von Hohenheim (Paracelso, ca. 1493-1541) revolucionó el mundo de la medicina al rebatir las creencias antiguas sobre la enfermedad y la sanación y afirmar que las enfermedades se debían a ciertos agentes externos ajenos al cuerpo a los que se podía atacar con la ayuda de determinadas sustancias químicas, por lo que se le considera el fundador de la Iatroquímica. Paracelso sostuvo la teoría de los "tres principios" que reducía toda la química a tres elementos fundamentales: azufre, mercurio y sal. Identificó al zinc como un metal único en 1526, como ya había hecho el metalúrgico hindú R. Samuccaya en el año 800 d.C., si bien sólo pudo ser aislado por A. S. Marggraf (1709-1782) en 1746.

La primera descripción de un procedimiento para aislar el antimonio elemental fue reflejada en 1540 por V. Biringuccio (1480-1539) en su libro titulado De la Pirotechnia (1540), donde también se incluye el primer relato escrito conocido sobre la práctica correcta de la fundición. Sin embargo, el sulfuro de antimonio(III) $\left(\mathrm{Sb}_{2} \mathrm{~S}_{3}\right)$ era conocido en Egipto como lápiz cosmético denominado "kohl" hacia el año 3100 a.C. y también se han encontrado piezas fabricadas de este material en Tello (actual Iraq) del año 40003000 a.C. (Kirk et al., 2007; Principe, 2013). El texto de Biringuccio de 1540 precedió al más conocido De re metallica escrito por G. Agrícola y publicado en 1556, el cual ha sido incorrectamente acreditado como el descubridor del antimonio metálico. Pero la autoría de este descubrimiento no está exenta de polémica, porque el libro El carro triunfal del antimonio describe en 1646 la preparación del antimonio metálico, supuestamente escrito por el monje Basilio Valentín hacia 1450; si esta noticia fuera auténtica la autoría del descubrimiento dejaría de pertenecer a Biringuccio, aunque ya en 1710 W. G. F. von Leibniz (1646-1716) realizó una cuidadosa investigación con la que concluyó que no había existido ningún monje llamado "Basilius Valentinus" y que el autor del texto era su editor, J. Thölde ( $c a$. 1565-ca. 1624), versión que es la aceptada hoy en día (Priesner \& Figala, 1998). Otro elemento cuyo descubrimiento se ha atribuido a Valentín es el Bismuto, que ya había sido citado en 1574 como muestra la figura 1, pero que no fue identificado definitivamente hasta 1753 por C. F. Geoffroy (1729-1753).

La llegada de los europeos a América supuso un importante impulso para el desarrollo de las ciencias y las técnicas en el viejo continente. Europa necesitaba barcos robustos que pudiesen atravesar el Océano y que navegasen con técnicas de navegación de altura, expertos que analizasen la inmensa cantidad de nuevas especies que se encontraban a cada paso y personas cualificadas que aplicasen sus conocimientos a la explotación de los yacimientos americanos de metales preciosos, que fue uno de los principales motores del desarrollo técnico y científico en la España del Renacimiento. La amplia serie de innovaciones que se inició con el novedoso método de amalgamación de minas de plata de Bartolomé de Medina en 1555 culminó con la publicación en 1640 del Arte de los metales de Álvaro Alonso Barba (ca. 1569-1662), tratado que expuso las nuevas metodologías descubiertas por su autor junto con las de otros metalurgistas. Esta obra tuvo una enorme difusión porque se imprimieron varias ediciones en castellano y se hicieron traducciones al inglés, alemán y francés (Castillo Martos, 2006).

La importancia económica que la determinación de la ley de las monedas tuvo en la España renacentista motivó que en sus cecas se instalaran los mejores medios técnicos de la época para el análisis químico cuantitativo. La principal figura en este campo fue el leonés Juan de Arfe Villafañe (1535-1603), "ensayador" de la ceca de Segovia y autor del Quilatador de plata, oro y piedras de 1572, primer tratado sobre el tema impreso en Europa. Además, el laboratorio de destilación más importante de la Europa renacentista se localizó en El Escorial, donde Diego de Santiago (s. XVI) fue el más destacado de los "destiladores de Su Majestad" que trabajaron en él. De Santiago publicó en 1598 el libro Arte separatoria, en el que 


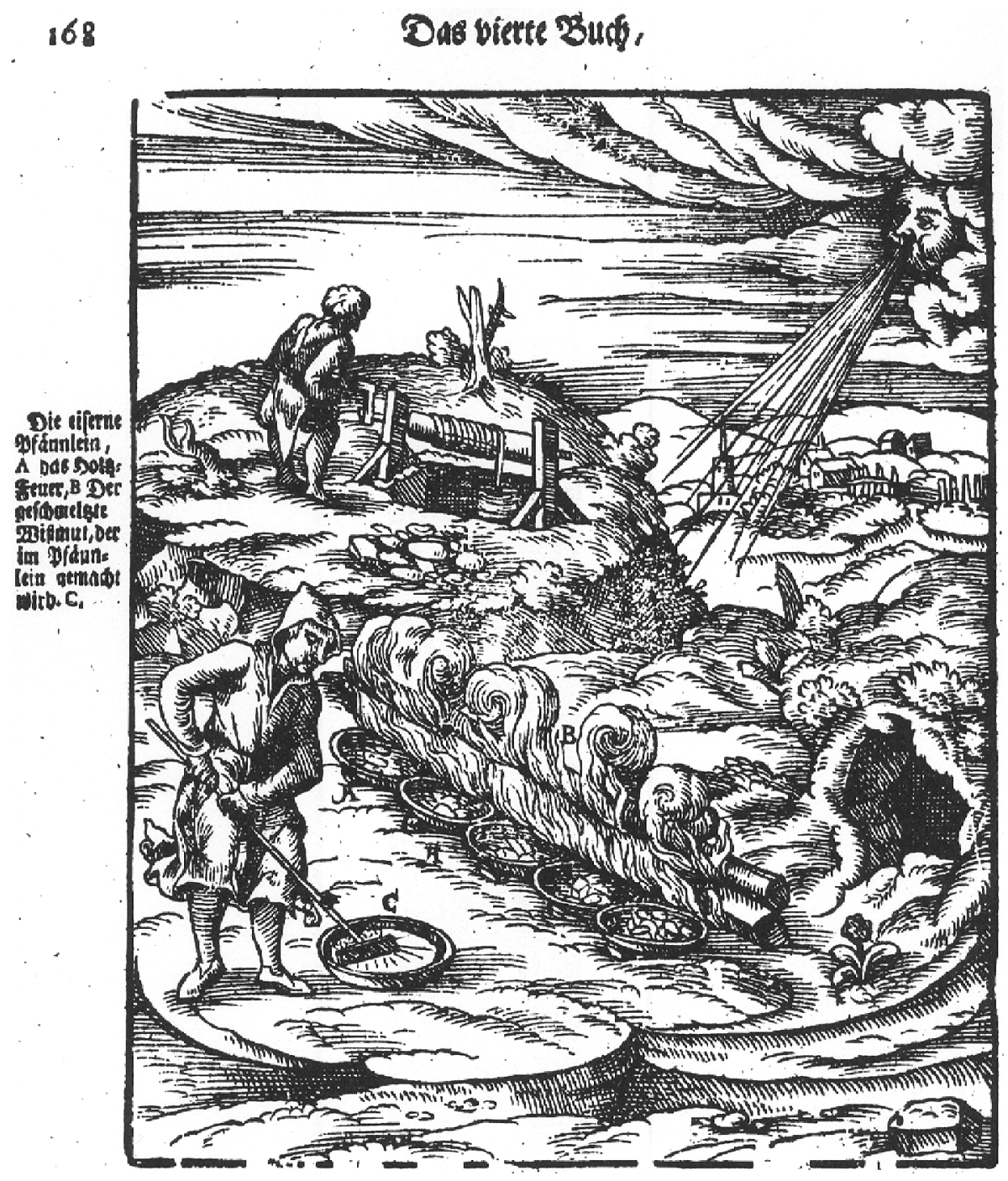

Fig. 1.-Obtención del bismuto en el siglo XVI (Erker, 1736).

describió un "destilatorio de vapor" de su invención que incluía unas enormes torres de destilación (López Piñero, 1979).

\section{El tránsito del Renacimiento a la llustración}

El final del siglo XVI y el comienzo del siglo XVII contempló el trabajo de A. Libavius (15501616), quien presentó la química como un conjunto de prácticas que poseían una metodología perfectamente sistematizada para su ejecución y realizó importantes aportaciones como los usos del cloruro de estaño, que se conoce como "licor de Libavius". Este alquimista escribió en 1597 un libro sistemático de química titulado Alchemia, que incluía instrucciones para la preparación de diversos ácidos fuertes y publicó algunas de sus obras bajo el seudónimo de Basilius de Varna (UAB, 2011).

La invención de la imprenta contribuyó de forma decisiva a la rapidez de la propagación de todo tipo de conocimientos, entre los que por supuesto se encontraban los saberes alquímicos. Una conocida compilación de estos tratados fue el Musaeum hermeticum (1625), que tuvo gran popularidad y cuyo propósito era presentar una colección representativa de escritos alquímicos relativamente breves. Se considera que fue un suplemento a las grandes enciclopedias alquímicas como el Theatrum chemicum 
(1602-1661) o la Bibliotheca Chemica Curiosa (1702). El Musaeum representa una forma diferente de entender la alquimia, menos oscura que las obras de los antiguos maestros. Está formado por varios capítulos, escritos cada uno por un autor diferente, entre los que destaca el firmado por Basilio Valentín titulado las "Doce Claves", que si se leen con nuestros ojos del siglo veintiuno resultan totalmente indescifrables. Por ejemplo, en la "Primera Clave" se detalla el siguiente texto: "Permite que la diadema del rey sea de oro puro, y haz que la reina, que está unida a él en matrimonio, sea casta e inmaculada. Toma un lobo gris y fiero, de los que encuentras en los valles y montañas del mundo por donde vaga salvaje y hambriento. Júntalo con el rey $\mathrm{y}$, cuando lo haya devorado, calcínalos en el fuego hasta reducirlos a cenizas. Una vez que hayas realizado este proceso tres veces, el rey estará liberado". Este párrafo se ilustra con el grabado reproducido en la figura 2.

Esta imagen ha resultado indescifrable hasta hace poco, pero hoy en día se entiende mejor su significado. Para ello se han realizado análisis muy completos de los crisoles y escarificadores encontrados en Austria y en otros lugares (Martinón-Torres, 2003 y 2008). Estos resultados se han comparado con los libros de alquimia y así se ha podido "traducir" el texto de la Primera Clave de Valentín, encontrándole un significado nada esotérico. En la literatura alquímica, el antimonio era denominado "lupus metallorum", tanto por el aspecto que tiene la antimonita (estibina, $\mathrm{Sb}_{2} \mathrm{~S}_{3}$ ) muy similar al pelaje de un lobo "gris y fiero", como porque era capaz de "disolver" otros metales como el oro o la plata. El rey representa el oro purificado gracias a la acción del antimonio, y la reina es la plata; el anciano de la pierna de madera simboliza Saturno, que en la literatura alquímica designaba al plomo que podía facilitar la refinación de la plata en la copela donde se muestra la bolita de dicho metal. En el siglo veintiuno, en vez de dibujar este grabado escribiríamos (Greenberg, 2007):

Oro impuro + Estibina $\rightarrow$ Aleación de oro y antimonio + Sulfuro de plata + Sulfuro de cobre:

$2(\mathrm{Au}+\mathrm{Ag}+\mathrm{Cu})+\mathrm{Sb}_{2} \mathrm{~S}_{3} \rightarrow 2 \mathrm{Au} / \mathrm{Sb}+\mathrm{Ag}_{2} \mathrm{~S}+2 \mathrm{CuS}$

La aleación de oro y antimonio es "purificada" al ser sometida al calor del fuego en el crisol en tres

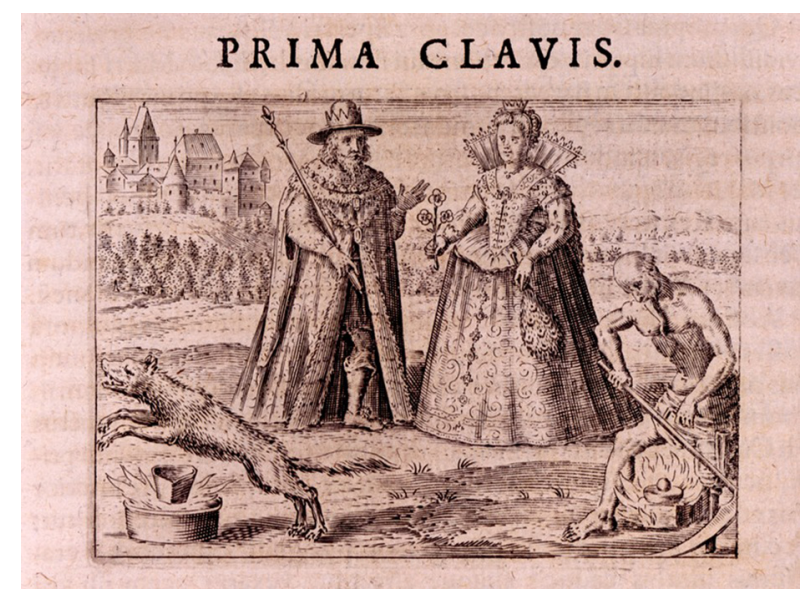

Fig. 2.-Prima Clavis, la Primera Llave, grabado realizado por M. Merian (1593-1650). Fuente: Musaeum hermeticum, 1678, 373-432.

ocasiones, hecho que se representa por las tres flores que lleva la reina en la mano. Nosotros lo escribiríamos como:

$$
4 \mathrm{Au} / \mathrm{Sb}+3 \mathrm{O}_{2} \rightarrow 4 \mathrm{Au}+2 \mathrm{Sb}_{2} \mathrm{O}_{3} \text { (vapor) }
$$

Los alquimistas de la segunda mitad del siglo XVII realizaron algunos importantes hallazgos, ${ }^{1}$ entre los que destaca el conseguido en 1669 por $\mathrm{H}$. Brandt (1630-1692) quien, en el curso de sus experiencias con la orina, obtuvo una sustancia blanca y cérea que resplandecía en la oscuridad, convirtiéndose en el primer descubridor reconocido de un elemento químico: el fósforo. Brandt fue inmortalizado por J. W. de Derby en su pintura "El alquimista en busca de la piedra filosofal", título engañoso que camufla la auténtica realidad científica que refleja dicho cuadro (Emsley, 2000).

Otra gran figura de la segunda generación de paracelsistas que desarrolló su actividad durante la segunda mitad del siglo XVII fue J. B. van Helmont (1577-1644), quien realizó, entre otras, importantes

${ }^{1}$ Para saber más sobre la alquimia se puede consultar la revista francesa Chrysopoeia de la Société d'Étude de l'Histoire de l'Alchimie, la revista británica Ambix de la Society for the History of Alchemy and Chemistry, y la revista electrónica Azogue (http://www.revistaazogue.com/, visitada el 27.II.2014) dedicada al estudio histórico-crítico de la alquimia. Está escrita en castellano y presenta estudios rigurosos sobre esta disciplina que dan una visión moderna y seria de ella. Asimismo es de obligada consulta el fondo bibliográfico que está recuperando el CSIC a través del proyecto de investigación dirigido por J. Pérez Pariente (http:// catalogochymico.icp.csic.es/index.jsp, visitada el 27.II.2014; Pérez Pariente, 2005 y 2010). 
investigaciones sobre los gases y los hidróxidos, creando el término "gas" y denominando "álcalis" a las lejías. Sus obras completas fueron publicadas en 1648 por su hijo con el título Ortus Medicinae. En esencia, el objetivo último de los experimentadores del s. XVII era establecer un sistema universal completo a la manera de los filósofos griegos. Tomaron de sus ideas las que parecían aplicables a las reacciones químicas y elaboraron así una nueva química "mecánica", a la que hay que agradecer el destierro de toda clase de fuerzas ocultas en las propiedades de las sustancias. A lo largo del siglo XVII se efectuaron numerosas experiencias que demostraron la posibilidad de generar el vacío y se desarrolló el microscopio, instrumento que evidenció la discontinuidad de la materia.

También hay que destacar las contribuciones de R. Boyle (1627-1691), quien propuso que la química merecía ser estudiada en sí misma sin ser sólo una ciencia auxiliar de la medicina y la farmacia. Gracias a un riguroso método experimental, Boyle demostró que los cuatro elementos de Aristóteles y los tres principios de los alquimistas (mercurio, azufre y sal) no merecían ser llamados elementos o principios. Sin embargo, en el siglo XVII el concepto de elemento era prácticamente el mismo que el establecido por Aristóteles: un elemento era el componente fundamental de toda la materia. Así, si el azufre era considerado un elemento, formaba parte tanto del oro como del mosto de la uva. En su libro The Sceptical Chymist (1661), Boyle se cuestionó esta idea de elemento químico, preguntándose si no existirían más elementos que los cuatro considerados en ese momento.

\section{Las luces de la llustración que iluminaron el camino}

El final del siglo XVII y la entrada en el XVIII contempló importantes contribuciones en el campo de la ciencia y la técnica, porque gracias a las aportaciones de I. Newton (1642-1727) entre otros, se interpretaron los fenómenos naturales de acuerdo con un modelo mecánico, sencillo y razonado. La química estaba constituida por un conjunto de prácticas que permitían obtener numerosos productos, pero el gran fallo que presentaban las diferentes teorías químicas de esta época era la ausencia de la noción de cuerpo simple, carencia que impedía la correcta clasificación de las sustancias y favorecía las organizaciones arbitrarias. Esta idea se ilustra en la figura 3, donde se muestra una tabla de "caracteres chymicos" con los símbolos empleados por N. Lémery (1645-1715), en la que se puede apreciar que se entremezclaban elementos (azufre, oro, mercurio ...), compuestos (agua, cal, cinabrio ...), aparatos de laboratorio (crisol), operaciones químicas (sublimar), épocas del año (capricornio, aries ...) y fuentes de energía (fuego).

En el siglo XVIII o "Ilustración" se creía que, gracias al conocimiento, la humanidad se libraría de las tinieblas de la ignorancia y la superstición. El sustrato que conformaba la mentalidad ilustrada estaba constituido por la revolución científica producida en el siglo XVII, unido al desarrollo de las ciencias experimentales generado a partir de ella. Con la Ilustración aumentó de forma espectacular la aplicación de la ciencia a la técnica y a la fabricación artesana y viceversa, de forma que este fenómeno, a la par que modificaba el estilo de vida de gran número de personas, generaba jugosos beneficios. Por ello, los diferentes gobiernos de esta época impulsaron la creación de instituciones donde la ciencia tuvo un destacado protagonismo y así se generó la necesidad de un personal cualificado que adquirió prestigio y relevancia social. Paralelamente se produjo la necesidad de intercomunicación entre ellos, de manera que surgieron numerosas publicaciones que fueron el vehículo perfecto para la transmisión de las ideas y teorías nacidas durante esta centuria. Una de ellas fue la Encyclopédie editada por Diderot \& D'Alembert desde 1751 hasta 1772 , obra monumental estructurada de forma alfabética por temas, que se convirtió en el modo de expresión de las ideas ilustradas y reflejó la situación de los conocimientos de la época. Como interpretaba el mundo desde un punto de vista racional, su publicación produjo el rechazo del clero y de los gobiernos tradicionales, así como bastantes discusiones entre sus partidarios y sus detractores. Fue continuada por la Encyclopédie Méthodique o Encyclopédie Panckoucke desde 1782 hasta 1832, en la que participaron un gran número de autores en 210 volúmenes. 
Explicacion de los mas Comunes Caracteres Chymicos.

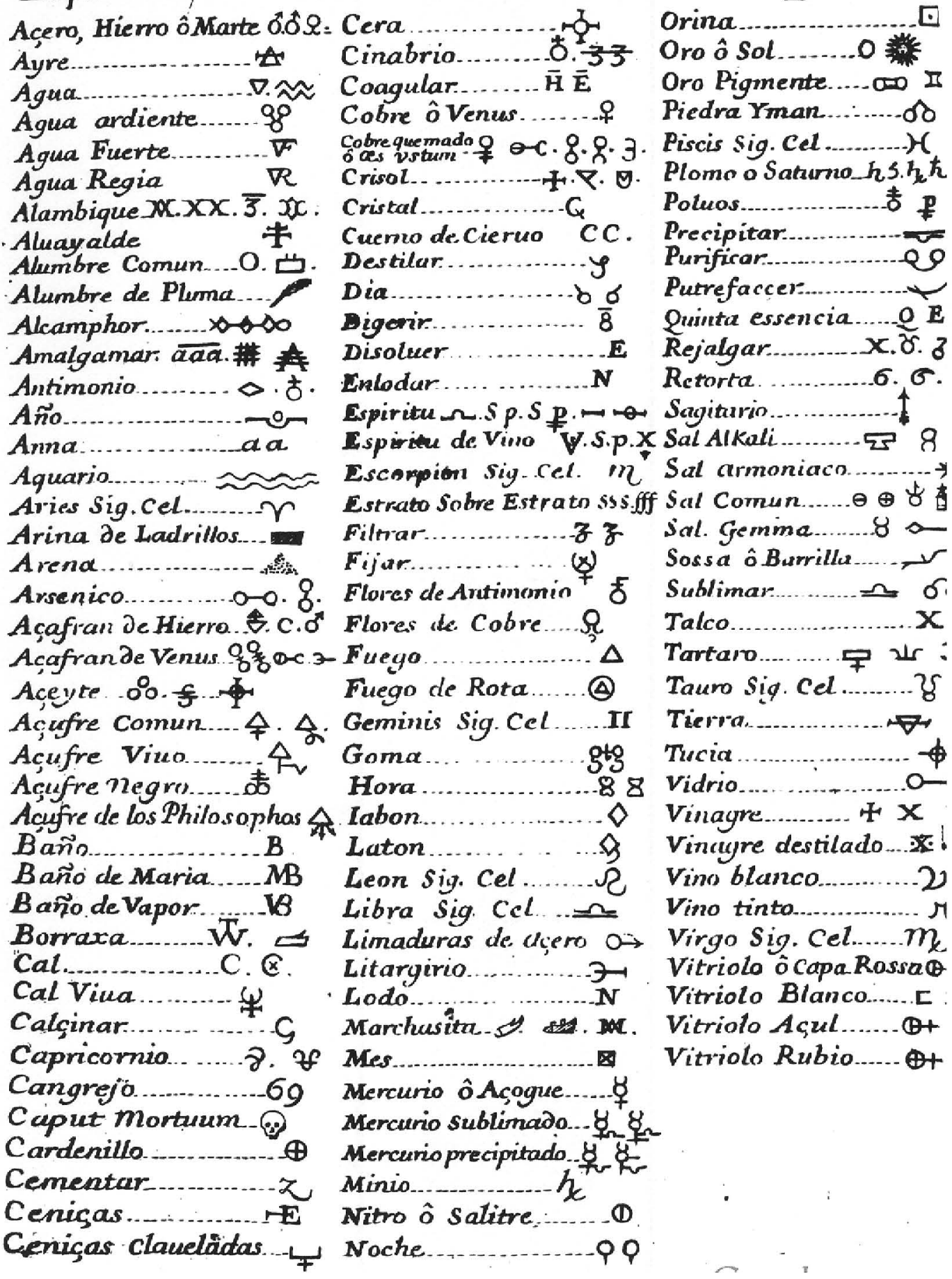

Fig. 3.-Tabla con símbolos empleados en las obras de química de principios del siglo XVIII. (Lémery, 1703).

A medida que los métodos experimentales se perfeccionaron se pudieron reconocer y aislar más elementos químicos, de manera que G. Brandt demostró en 1732-35 que el color azul del vidrio se debía a un nuevo tipo de metal y no al bismuto como se pensaba anteriormente. Sospechando que era el mismo 
material que formaba parte de un mineral azul obtenido en las minas del cobre alemanas que no contenía cobre, lo calentó con carbón vegetal, lo redujo y obtuvo cobalto puro.

Un caso particular ocurrió con el platino, metal que ya era conocido por los habitantes de la América precolombina. Sin embargo, la primera referencia europea sobre él se encuentra en los escritos del humanista italiano J. C. Escalígero (1484-1558) como una descripción de un metal misterioso "hasta ahora de imposible fusión por cualquiera de las artes españolas" (Weeks, 1932), frase que refleja el alto nivel de las técnicas metalúrgicas en nuestro país. Pasaron los siglos y el marino ilustrado sevillano Antonio de Ulloa (1716-1795) se trasladó a la selva del Chocó (Colombia) en 1735 para medir un arco del meridiano, formando parte de una expedición geodésica auspiciada por la Academia de Ciencias de París. Allí observó que ciertos yacimientos habían sido desechados por contener un metal tan duro que no se alteraba por la calcinación, tal y como lo explicó en la crónica de su viaje. Lo denominó 'platina' porque los mineros lo llamaban 'plata de la mala', y los medios de esa época no conseguían romperla ni calcinarla, ni por supuesto extraer el metal que contenía. Después de numerosas peripecias en el viaje de vuelta a la península, Ulloa publicó sus hallazgos en 1748. A pesar de ello y de que $\mathrm{Ch}$. Wood (1702-1774) también había investigado el metal en 1741 , muchos textos consideran que la primera referencia escrita de que el platino era un nuevo metal fue hecha por W. Brownrigg (1711-1800) en 1750 (McDonald \& Hunt, 1982; Castillo, 2005).

Pero no fue ésta la única gloria que otorgó el platino a nuestro país, porque este metal sólo pudo malearse por primera vez gracias al perfecto trabajo de investigación que realizó el químico francés $F$. Chabaneau (1754-1842) en el Seminario de la villa guipuzcoana de Bergara, donde era profesor (Gago \& Pellón, 1994).

Por aquella época hubo otros metales que también fueron reconocidos como tales, como el ya citado zinc o el níquel, obtenido por A. F. Cronstedt (17221765) en 1751 cuando intentaba extraer cobre del mineral hoy conocido como "falso cobre" (arseniuro de níquel, NiAs). También J. Black (1728-1799) observó en 1755 que la sustancia llamada magnesia alba $(\mathrm{MgO})$ no era cal viva $(\mathrm{CaO})$ y así reconoció el magnesio, metal que sólo pudo ser aislado por H. Davy (1778-1829) con métodos electroquímicos en 1808.

Se puede observar que hasta ahora todos los elementos identificados eran sólidos, pero a lo largo de la segunda mitad del siglo XVIII se desarrolló en Europa la denominada "química neumática", cuyo principal objetivo era recoger e identificar los gases producidos en las reacciones. En esta tarea destacaron los químicos ingleses, sobre todo J. Priestley (17331804), gracias a cuyas investigaciones $H$. Cavendish (1731-1810) fue capaz de distinguir el hidrógeno de otros gases en 1766, aunque previamente Paracelso, Boyle y Priestley habían observado su producción cuando reaccionaban ácidos fuertes con metales. Fue denominado con varios nombres hasta que A. L. Lavoisier (1743-1794) le asignó el nombre con el que actualmente lo conocemos. Otros elementos gaseosos obtenidos en el siglo XVIII fueron el oxígeno (1771), el nitrógeno (1772) y el cloro (1774).

Otro caso particular fue el del manganeso. En el siglo XVII, el químico alemán J. R. Glauber (ca.1604-1670) produjo por primera vez permanganato, y a mediados del siglo XVIII el dióxido de manganeso se empleaba para la producción de cloro. Scheele fue el primero en describir que el manganeso era un elemento, pero fue J. G. Gahn (1745-1818) quien lo aisló por reducción del dióxido con carbono en 1774, unos años después de los experimentos realizados en Viena por I. G. Kaim (1746-1778) descritos en su tesis doctoral titulada "Dissertatio de metallis dubiis" (1770) y que, a pesar de su escasa difusión, le confirman como el primer científico en aislar el manganeso.

Scheele reconoció al molibdeno como un constituyente de la molibdenita en 1778 , pero no fue aislado hasta 1781 por P. J. Hjelm (1746-1813). Un año más tarde, M. von Reichenstein (1740 o 1742-1825 o 1826) observó la presencia de un nuevo metal en las menas de oro de Transilvania (1782), pero fue M. H. Klaproth (1743-1817) quien consiguió aislarlo por primera vez en 1798 y le asignó el nombre de teluro.

1783 fue un año de especial importancia para la ciencia española, porque los hermanos Juan José (1754-1796) y Fausto (1755-1833) de Elhuyar consiguieron aislar el wolframio en el laboratorio químico del ya citado Seminario de Bergara, situado en 
la denominada "Casa de Zabala", muy próxima al centro docente (Pellón, 2013).

A pesar del nombre que le dieron sus descubridores, este elemento químico es denominado hoy en día de dos maneras diferentes: Wolframio y Tungsteno. Los hermanos Elhuyar partieron del mineral denominado wolframita (espuma o baba de lobo en alemán) y de ahí le asignaron su nombre, pero como en 1758 Cronstedt lo denominó Tungsteno ("piedra pesada" en sueco), en el mundo anglosajón fue adoptado el nombre de Tungsten en lugar de Wolframium a pesar que el símbolo químico es W. ${ }^{2}$ Pero quien generalizó el nombre de Tungsteno fue Lavoisier, el protagonista indiscutible de la química de esta época, quien supo aprovechar todos los avances existentes y aplicó de forma rigurosa el método experimental para obtener deslumbrantes éxitos científicos. Aunque desarrolló a lo largo de su vida una muy variada gama de actividades, fue su contribución a los progresos de la química lo que le ha proporcionado fama inmortal. El triunfo de su doctrina no consistió solamente en la lucha de un pequeño grupo de adeptos perfectamente entrenados contra el flogisto, sino que, ayudado por el prestigio y la publicidad que le aseguraba la Academia de Ciencias de París, cultivó un amplio espectro de materias. En todos los campos en los que trabajó aplicó los principales ideales ilustrados: derrotó a la tradición, normalizó el lenguaje, explotó los recursos de la métrica e hizo construir costosos instrumentos para poner de su lado a la evidencia experimental. Lavoisier demostró cómo el oxígeno formaba parte de todos los ácidos conocidos en ese momento, por lo que le asignó ese nombre. Su intervención modificó la tradición del flogisto y de los elementos / principios, pero dejó libres otras muchas áreas que se desarrollaron gracias al trabajo de otros investigadores, como el estudio de las sales realizado por C. F. Wenzel (1740-1793) y J. B. Richter (1762-1807) o la química newtoniana de las afinidades que acometió Cl. L. Berthollet (1748-1822). Es decir, abrió el camino para los investigadores

\footnotetext{
$\overline{{ }^{2} \mathrm{El} \text { nombre wolframio }}$ fue oficialmente adoptado por la IUPAC en su $15^{\mathrm{a}}$ conferencia celebrada en Ámsterdam en 1949, pero fue suprimido en favor de Tungsteno en 2005 (IUPAC, 2005) a pesar de que los miembros hispanohablantes de la IUPAC, entre otros, impugnaron esta recomendación y abogaron por la adopción del nombre de Wolfram en inglés y su correspondiente Volframio en español, acorde con el símbolo químico que lo representa.
}

que le sucedieron, porque supo captar y canalizar los diferentes cursos de conocimientos que conformaban la Química. Su genialidad consistió sobre todo en hacer que esta ciencia, que después de Stahl avanzaba por un terreno sin fronteras entre los tres reinos de la naturaleza -animal, mineral y vegetal-, encontrara un territorio propio en el laboratorio, que se convirtió en el hábitat natural del químico.

Lavoisier también demostró que el agua era una sustancia compuesta y no un "elemento" simple, al realizar una experiencia espectacular en febrero de 1785 que duró dos días y consistió en un doble experimento: la síntesis y la descomposición del agua. Con él desterró definitivamente la teoría de los cuatro elementos, pero no contento con eso, reformó la nomenclatura y puso a la docencia de la química en la picota. Junto con L. B. Guyton de Morveau (1737-1816), Cl. L. Berthollet y A. F. Fourcroy (1755-1809) publicó el Méthode de nomenclature chimique (Cuchet, París, 1787), en el que reformaron la nomenclatura química utilizada hasta ese momento. Las propuestas de los cuatro geniales químicos estaban basadas en las ideas del filósofo É. B. de Condillac (1715-1780), para quien el lenguaje bien estructurado tenía una importancia fundamental en el progreso de la ciencia, porque era lo que facilitaba una ciencia bien hecha. De acuerdo con esta concepción, las sustancias más sencillas eran las que debían nombrarse en primer lugar, pero con nombres que recordaran las sensaciones que proporcionaban los objetos, según la cadena:

Objeto real $\rightarrow$ Sensación $\rightarrow$ Idea $\rightarrow$ Palabra (Lenguaje) $\rightarrow$ Conocimiento

La edición de esta obra marcó un momento crucial en la historia de la química, porque esta nomenclatura ha permanecido hasta nuestros días y gracias a ella, los químicos podemos comunicarnos entre nosotros con un lenguaje sistemático y exacto. Lamentablemente, este magnífico texto fue el que generalizó el término Tungsteno en lugar de Wolframio, como puede apreciarse en la figura 4.

Dos años después, mientras el pueblo francés tomaba La Bastilla, Lavoisier publicó el Tratado elemental de química (Cuchet, París, 1789), que presentaba de forma íntegra y sencilla las bases de su nueva química y contrariamente a lo que solía ser habitual en sus trabajos, estaba dirigida a "los principiantes" y no a los eruditos. Lavoisier proponía avanzar de lo conocido hacia lo desconocido, es decir, de 
108 N OMENCLATURE Noms. anciens. Noms nouvedux. Acide arfénical. Acide benżoniquẹ. Acide boracin. Acide charbonneuse. Acide citronien. Acide crayeux. Acide des fourmis. Acide dés pommes. Acide du benjoin. Acide du fel. Acide du foufre. Acide du fuccin. Acide du fucre. Acide du fuif. Aide du' vináigre. Acide du Wolfram, de) MM. Delhuyar. Acide fluorique. Acido formicin. Acide galactique. Acide gallique. • Acide lignique. Acide lithiafique. Axide malufien. Acide marin. Acide marin déphlogif: iigué.
C F I M I Q U E.

43

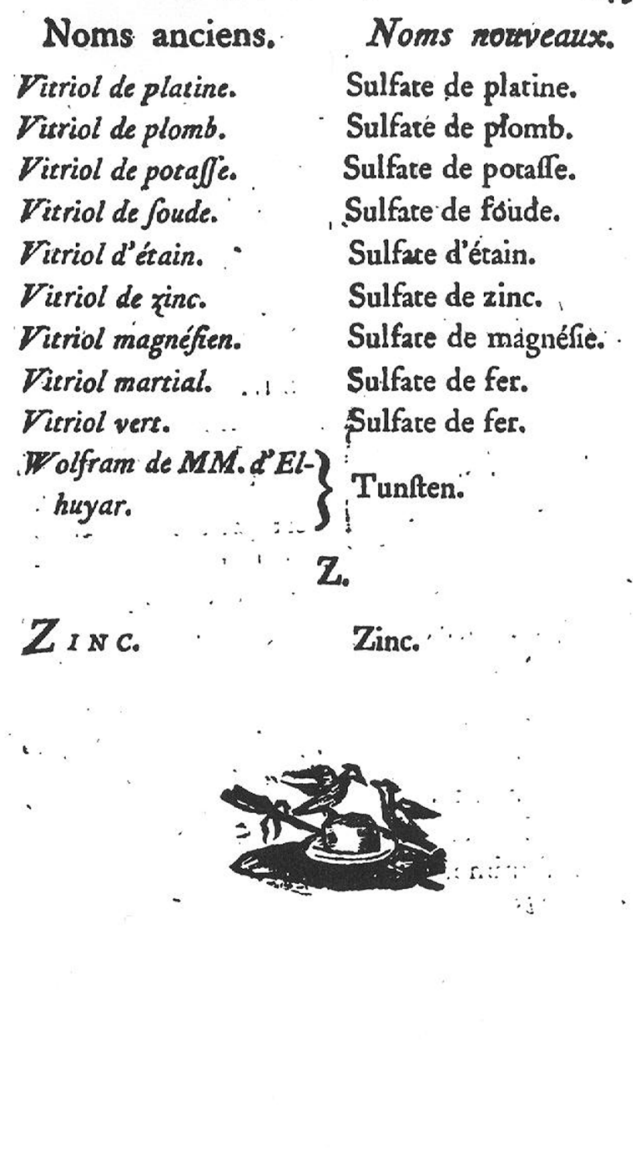

Fig. 4.-El "ácido del Wolframio o de MM. Delhuyar" según el nuevo método de nomenclatura química, y el "Wolfram de MM. Delhuyar" en el que puede apreciarse cómo se cambia el nombre del wolframio por el de tungsteno (Guyton de Morveau, 1787).

acuerdo con Condillac, "seguir la marcha natural de las ideas". Este texto contiene la que se considera la primera lista moderna de elementos químicos, que citaba los 23 elementos conocidos entonces más la luz, el calor y tres "radicales": "muriático", "fluórico" y "borácico", que más tarde derivarían en los elementos cloro, flúor y boro (Ver figura 5).

Gracias al trabajo de Lavoisier y sus contemporáneos, el siglo XVIII contempló el descubrimiento de varios elementos químicos desconocidos hasta el momento. Por eso en algunos casos suele haber confusión y polémica en la asignación de la gloria del descubrimiento, porque no es lo mismo sospechar la existencia de un nuevo elemento químico que llegar a identificarlo, conseguir aislarlo en estado impuro y por fin obtenerlo en estado puro. Los elementos "descubiertos" a finales del siglo XVIII fueron siete: estroncio (1787), zirconio y uranio (1789), titanio (1791), ytrio (1797), cromo (1797) y berilio (1798).

\section{Y de repente, se pudieron "pesar" los átomos}

El siglo XIX comenzó con el enunciado de la Teoría Atómica de Dalton (1803), que revolucionó el paradigma sobre la composición última de la materia. John Dalton (1766-1844) fue capaz de realizar algo que, durante muchos miles de años de evolución humana nadie había conseguido hasta ese momento: Dotar a los átomos de los elementos de una masa atómica relativa, como se muestra en la figura 6 . $\mathrm{Su}$ teoría contenía varias imprecisiones y errores, pero ofreció a los químicos del siglo XIX conceptos nuevos y muy importantes, que hicieron que causase 
D20 DES SUBSTANCES SIMPLES.
TABLEAU DES SUBSTANCES STMPLES

\begin{tabular}{|c|c|c|}
\hline & Noms nouveaux. & Noms anciens correfpandans. \\
\hline $\begin{array}{l}\text { Subftances fim- } \\
\text { ples qui appar- } \\
\text { tiennent aux } \\
\text { trois règnes \& } \\
\text { qu'on peut regar- } \\
\text { der comme les } \\
\text { elémens des } \\
\text { corpe. }\end{array}$ & 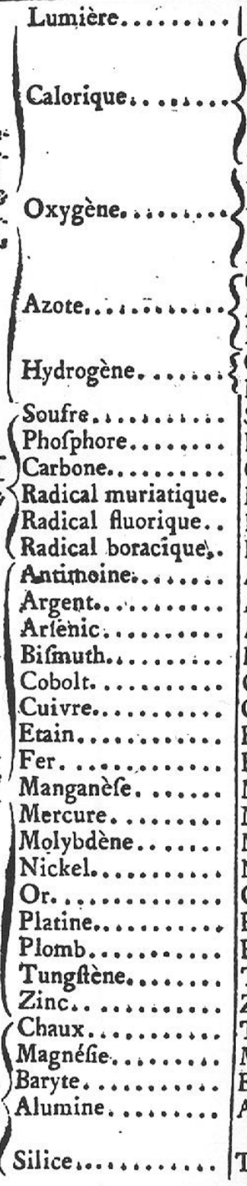 & 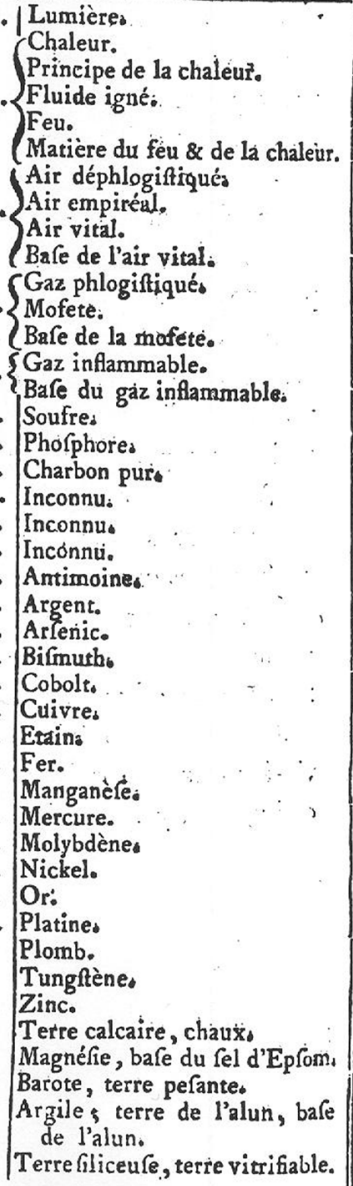 \\
\hline
\end{tabular}

Fig. 5.-Lista de los "elementos" químicos conocidos a finales del siglo XVIII. En ella ya no aparece la referencia a los hermanos Delhuyar (Lavoisier, 1789).

una auténtica conmoción entre sus colegas. Esta teoría cuantificó la vaga idea que se tenía de los átomos, concretó el concepto de elemento, explicó la discontinuidad de las proporciones dentro de los compuestos según las leyes de las proporciones constantes y múltiples y sugirió que la disposición de los átomos en un compuesto podía representarse esquemáticamente para que reflejase su estructura real (Pellón, 2012).

Las ideas de Dalton se difundieron a través de la comunidad científica, que ahora contemplaba el desarrollo de las operaciones químicas desde un punto de vista diferente. El mecanismo de las reacciones se explicaba a la luz de un nuevo paradigma,

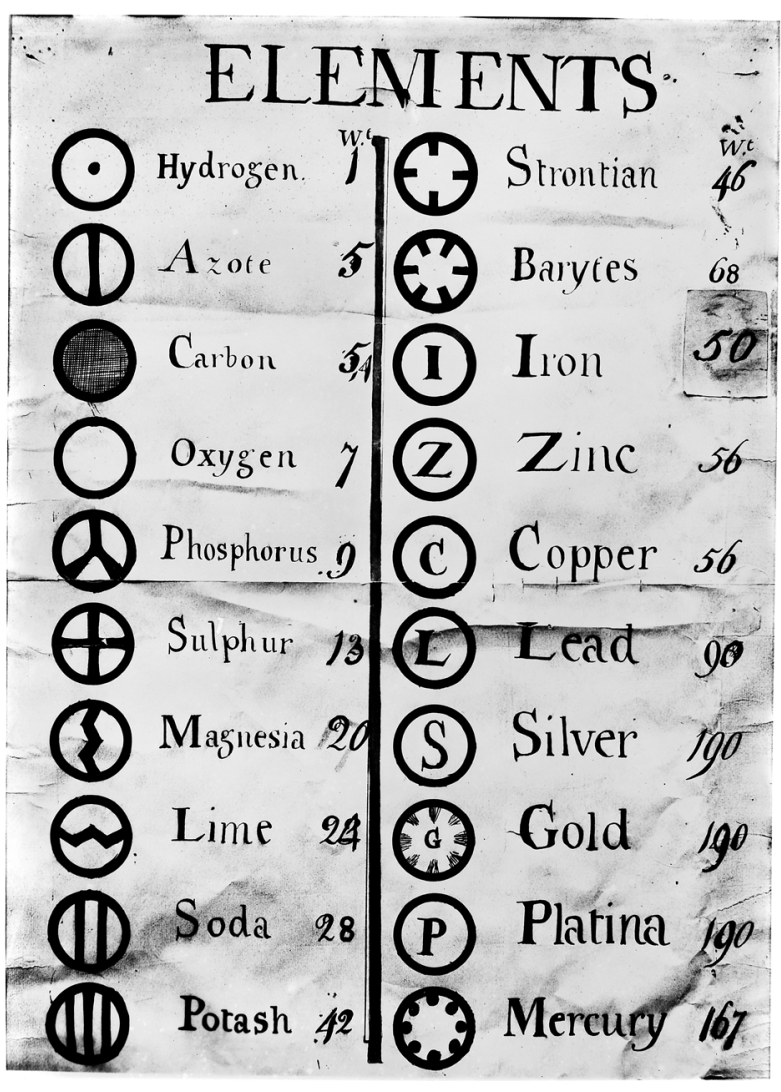

Fig. 6.-Lámina ilustrativa de los símbolos atómicos y masas atómicas relativas de los elementos químicos utilizada por $\mathrm{J}$. Dalton en sus lecciones (1806). (http://wellcomelibrary.org/, visitada el 26.II.2014).

que el mismo Dalton reconocía como "un nuevo sistema de filosofía química". Esta interpretación de los fenómenos originó discordancias donde antes no existían, y los investigadores se vieron obligados a solucionar varios problemas importantes para conseguir que el saber progresase y se estabilizase como "ciencia normal". Pero no fue tarea fácil, porque las numerosas inconsistencias y anomalías hicieron que, iniciado el segundo tercio del siglo XIX, aún permanecieran sin resolver dos cuestiones fundamentales: "¿Cuántos átomos hay en una molécula?"; y este número, "¿es el mismo para todos los elementos?". De este modo, al comenzar la cuarta década del siglo XIX, la comunidad científica era una torre de Babel y la anarquía era tan grande que una misma sustancia podía escribirse de muchas maneras; el ejemplo más representativo era el del ácido acético, para el que llegaron a existir 19 fórmulas diferentes. Era urgente hacer algo para remediar esta situación y 
F. A. Kekulé (1829-1896) propuso a su colega Ch. A. Wurtz (1817-1884) organizar una convención internacional para aclarar los conceptos de átomo, molécula y equivalente. Se reunieron del 3 al 5 de septiembre de 1860 en Karlsruhe (Alemania), en el que fue el primer congreso internacional de química que se celebró en el mundo. Participaron 140 químicos de varios países, siendo el único español presente el catedrático de la Universidad de Madrid Ramón Torres Muñoz de Luna (1822-1890) (Pellón \& Bilbao-Goyoaga, 2013). Durante esos tres días, los químicos discutieron entre sí sin ponerse de acuerdo, resultado lógico porque no parece muy apropiado decidir la naturaleza de los átomos y de las moléculas mediante un voto en una asamblea. Cuando finalizó el congreso se repartió entre los asistentes un folleto escrito por S. Cannizaro (1826-1910) titulado Sunto di un corso di Filosofía Chimica (Cannizaro, 2009). Después de leerlo en el viaje de regreso a su casa, J. L. Meyer (1830-1895) afirmó: "las escamas se cayeron de mis ojos, las dudas desaparecieron, y un sentimiento de calma ocupó su lugar". El Sunto, que ya había sido publicado en 1858, mostraba que la aplicación rigurosa de la hipótesis de Avogadro podía deshacer todas las incongruencias. A pesar de ello, la solución al conflicto no resultó inmediata, sino que fue necesario el advenimiento de otra generación para que la mayoría de los químicos europeos se pusieran de acuerdo en un único sistema de masas atómicas, cuyas bases fueron suministradas por la química orgánica.

Previamente, Berzelius había ideado en 1813 los símbolos que han llegado hasta nuestros días, y el madrileño Andrés Manuel Del Río (1764-1849) había examinado en 1801 unas muestras minerales procedentes de una mina situada en Zimapán (México) y había llegado a la conclusión de que contenían un elemento metálico desconocido hasta el momento. Como homenaje al lugar de su hallazgo lo denominó "Zimapanio", pero al observar la diversidad de colores que presentaban sus sales cambió su nombre por "Pancromio", que en griego significa "todos los colores". Poco después, al observar que sus sales calentadas cambiaban su color al rojo, volvió a cambiarlo por el de "Eritronio", que en griego significa "rojo". Un año más tarde envió unas muestras que contenían el nuevo elemento a A. von Humboldt (1769-1859), quién junto al químico francés H. V.
Collet-Descotils (1773-1815) las analizaron y declararon en 1805 -incorrectamente- que el nuevo elemento era sólo una muestra impura de cromo. Del Río aceptó sus resultados y se retractó de su afirmación, favoreciendo así que el químico sueco $\mathrm{N}$. G. Sefström (1787-1845) detectara en 1831 que los minerales de hierro con los que trabajaba contenían un elemento desconocido al que denominó Vanadio en honor a la diosa escandinava Vanadis, nombre que oficialmente mantiene hasta la fecha. Ese mismo año F. Wöhler confirmó los principios de la obra de Del Río intentando reparar la injusticia cometida, a la vez que el geólogo G.W. Featherstonhaugh (1780-1866) sugirió que el vanadio debería llamarse "Rionium" en su honor, sugerencia que no prosperó (Castillo, 2005; Weeks, 1932).

El aislamiento del vanadio fue tarea difícil, que no fue obtenido hasta que el químico H. E. Roscoe (1833-1915) los obtuvo en 1867 por la reducción del cloruro de vanadio(III) con hidrógeno.

Una vez iniciado el siglo XIX, los químicos se dedicaron a buscar nuevos elementos químicos y los resultados de su trabajo se produjeron en cascada, llegándose a reconocer 31 elementos nuevos desde 1801 hasta 1868. En esta tarea resultaron de especial ayuda los análisis espectrales y los métodos electroquímicos empleados por H. Davy, que sugirieron y confirmaron la existencia de nuevos elementos como el magnesio, bario, estroncio, calcio, sodio, potasio boro, cesio, rubidio, talio, indio o el gas noble helio. Gracias a estas técnicas el número de elementos químicos conocidos fue creciendo sin cesar y surgió la necesidad de ordenarlos de alguna manera. Al estudiar y comparar sus propiedades físicas y químicas se puso de manifiesto que existían semejanzas entre algunos de ellos, por lo que surgió la idea de agruparlos según sus propiedades.

\section{Ordenar el desorden}

El químico alemán J. Döbereiner (1780-1849) agrupó los elementos hasta entonces conocidos en series de tres denominadas "tríadas", de acuerdo con la similitud de sus propiedades químicas, que variaban gradualmente del primero al último. Además, el elemento central poseía un peso atómico (PA) aproximadamente igual a la semisuma de los PA de los elementos extremos. La clasificación propuesta 
por Döbereiner en 1817 fue completada por otros investigadores de forma que hacia 1850 se había llegado a identificar unas veinte triadas, pero esta clasificación se descartó ante las numerosas excepciones e irregularidades encontradas.

El siguiente intento de ordenación lo protagonizó el geólogo francés A-B. E. de Chancourtois (18201886) en 1862, quien propuso una clasificación de los elementos en forma de hélice que llamó "Caracol Telúrico". En un cilindro trazó una hélice con un ángulo de $45^{\circ}$ sobre la base y en ella fue colocando los elementos en función creciente de sus pesos atómicos, de tal manera que la línea vertical (generatriz) del cilindro interceptaba a los elementos con propiedades semejantes. Pronto se vio que este ordenamiento no se podía generalizar, por lo que el químico inglés J.A.R. Newlands (1838-1898) ordenó los elementos químicos entonces conocidos en grupos de siete, cada uno en función creciente a sus pesos atómicos, de tal modo que el octavo tenía propiedades semejantes al primer elemento del grupo anterior. Esta forma de clasificación se denominó "Ley de las Octavas", y aunque en un principio fue ridiculizada por sus contemporáneos en la Royal Chemical Society, hasta que 23 años después la misma entidad le otorgó el máximo reconocimiento debido a su importante contribución al desarrollo de la ley periódica de los elementos químicos.

En este momento las técnicas experimentales se habían desarrollado de tal manera que los pesos atómicos se pudieron determinar con un gran nivel de precisión, por lo que su estudio mostró que si se ordenaban los elementos según un criterio creciente de masas atómicas aparecía una periodicidad en la variación de sus propiedades físicas y químicas. Este hecho llevó a que el alemán L. Meyer y el ruso D. I. Mendeleev (1834-1907) establecieran en 1869 una ordenación de los 63 elementos conocidos hasta el momento por orden creciente de masa atómica en series de filas y columnas, en las que los elementos que estaban en la misma columna tenían propiedades físicas y químicas parecidas. Mendeleev también pudo prever la existencia de elementos aun no hallados, reservándoles el hueco que les correspondería en la tabla periódica (Román, 2008). Su idea fue completada con el establecimiento de una serie de pesos atómicos exactos y con la concepción del átomo con un núcleo en el que existían un número definido de protones con igual número de electrones que giran a su alrededor en una corteza electrónica.

Este ordenamiento condujo a que H. G. J. Moseley (1887-1915) estableciera el concepto de número atómico (Z) en 1912. A partir de este hallazgo se vio que las propiedades periódicas de los elementos no dependían de su masa atómica sino de su número atómico, que más tarde se comprobó que coincidía con el número de protones del núcleo. A partir de esta idea surgió el Sistema Periódico actual gracias al trabajo de A. Werner (1866-1919) y F. A. Paneth (1887-1958), en cuyas 18 columnas y 7 filas los elementos están distribuidos por un criterio creciente de número atómico, lo cual hace que haya tres parejas en las que un elemento está precedido por otro de masa atómica mayor, contradiciendo así lo propuesto por Mendeleev.

A lo largo del siglo veinte el desarrollo de la Química ha sido espectacular, completándose la especialización iniciada en el siglo XIX. Además, gracias al desarrollo de los Modelos atómicos y de la Mecánica cuántica se han podido completar los huecos que faltaban en la Tabla Periódica, por lo que hoy en día se han podido aislar todos los elementos que la conforman. El país que más elementos ha aislado ha sido Inglaterra (21), seguido de Suecia (19) y Alemania (19). A España se le reconocen dos (W y Pt), aunque hemos de reivindicar el vanadio descubierto por Andrés Manuel del Río.

Nombrar a las sustancias nunca ha sido una cuestión sencilla y esta tarea ha generado una fuerte controversia en varios casos. Aparte de los ya citados wolframio y vanadio, se puede comentar la dificultad que entrañó la designación de nombre para el niobio $(Z=41)$, iterbio $(Z=70)$ o lutecio $(Z=71)$. Todo comenzó al ser descubierto el mineral gadolinita o yterbita en 1800 por J. Gadolin (1760-1852), quien observó que estaba compuesta por tierras raras ricas en elementos lantánidos. Fueron denominadas también "tierras raras de Erbia" por estar cerca de la ciudad sueca de Ytterby y contenían los elementos Erbio, Iterbio, Terbio, Lutecio, Cerio y el propio Gadolinio. Estas también fueron estudiadas por J. Ch. Galissard de Marignac (1817-1894) en 1878, y en 1907, el químico francés G. Urbain (1872-1938) encontró en ellas dos elementos químicos, a los que denominó 
Lutecio $(Z=71)$ en honor a Lutecia, el nombre que recibió la ciudad de París en tiempos de Roma, y Neoiterbia $(Z=70)$. De forma independiente, $C$. A. von Welsbach (1858-1929) también hizo este mismo descubrimiento y los llamó Aldebaranio y Casiopeo en honor a la estrella Aldebarán y a la constelación de Casiopea. En 1909, la comisión de la Masa Atómica responsable en la atribución de nombres a los nuevos elementos químicos descubiertos denominó Iterbio $(\mathrm{Yb})$ al elemento $\mathrm{Z}=70 \mathrm{y}$ Lutecio $(\mathrm{Lu})$ al elemento $Z=71$.

El Einstenio (Es, $Z=99)$ y el Fermio (Fm, $Z=100)$ denominados así en honor de A. Einstein (18791955) y de E. Fermi (1901-1954) se descubrieron en diciembre de 1952 en los restos de la primera explosión termonuclear realizada un mes antes en el Pacífico. Cuando fueron propuestas las dos denominaciones no entraron en polémica porque Einstein y Fermi ya habían fallecido (Ghiorso, 1955).

El resto de los elementos transférmicos (con $\mathrm{Z}>100$ ) se han sintetizado artificialmente y a medida que se han ido descubriendo se han situado de izquierda a derecha en las casillas marcadas del séptimo periodo. Se llegó al acuerdo de que los nuevos elementos se denominarían con los nombres que eligieran sus descubridores, pero en el caso de los elementos 104 a 108 se produjo una virulenta controversia cuando varios investigadores de nacionalidades diferentes reivindicaron simultáneamente su descubrimiento en la década de 1960. Estos grupos fueron los norteamericanos del Lawrence Berkeley National Laboratory (LBNL, Berkeley, California), los rusos del Instituto Central de Investigaciones Nucleares (Dubna) y los alemanes del Instituto de investigación de iones pesados, Gesellschaft für Schwerionenforschung (GSI, Darmstadt).

El elemento 109 fue encontrado por casualidad en el GSI al bombardear bismuto-210 con núcleos acelerados de hierro-74 en 1982, hecho que demostró que las técnicas de fusión nuclear podían ser utilizadas para crear nuevos núcleos pesados. Su nombre Meitnerio (Mt) fue sugerido en honor a la matemática y física L. Meitner (1878-1968) (Thierfelder, 2008).

En 1999, los investigadores del LBNLde California anunciaron el descubrimiento del elemento 116 al observar el decaimiento- $\alpha$ de un átomo de mayor número atómico. Lo denominaron Livermorio en honor al lugar en el que está situado el laboratorio, pero el año siguiente se retractaron tras ser incapaces de repetir el experimento. En junio del año 2002 el director del laboratorio anunció que los datos obtenidos habían sido falseados por el investigador $\mathrm{V}$. Ninov, tras lo que se realizaron numerosas pruebas tanto en California como en Dubna para intentar obtener una muestra de dicho elemento, todas infructuosas hasta que en octubre de 2006 se demostró que el bombardeo de átomos de californio-249 con iones de calcio-48 produjo Ununoctio (elemento 118), que posteriormente decaía a Ununhexio en pocos

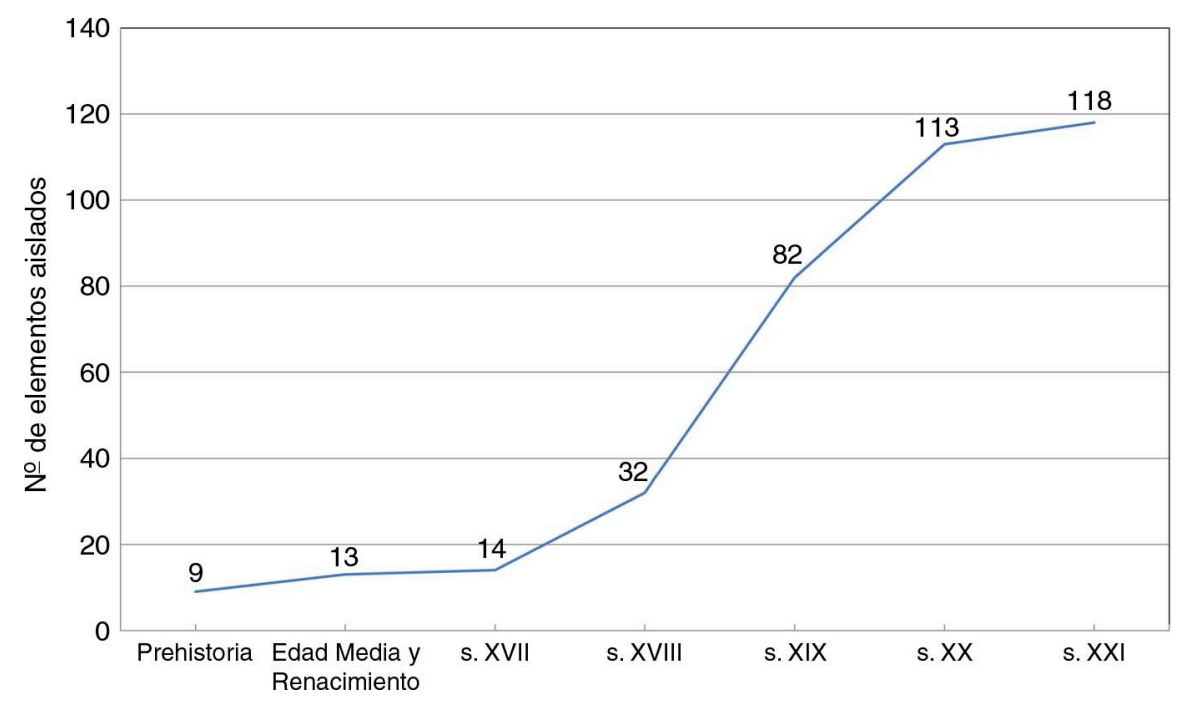

Fig. 7.-Gráfica que muestra la progresión del "descubrimiento" de los elementos químicos a lo largo de los años. 
milisegundos y que se había producido al menos en tres ocasiones (Morss et al., 2011).

\section{Conclusiones}

En este trabajo hemos realizado un viaje a vuelapluma por la ciencia y la técnica desde la Prehistoria hasta nuestros días, con el objetivo de enmarcar el descubrimiento de los elementos químicos, en el que hay que destacar cómo las bases suministradas por la alquimia, el siglo XVIII y el esplendor de la Ilustración llevó a la Encyclopédie, al genial Lavoisier y a una "revolución anunciada" que, entre otras muchas cosas, generó los inicios del periodismo científico. El siglo XIX contempló cómo gracias a Dalton se pudieron "pesar" los átomos, así como el "nacimiento" de las especialidades en Química que llevó a que en el siglo XX se alcanzase la madurez de esta ciencia. Desde esta perspectiva global, resulta inevitable considerar que lo que denominamos Alquimia tuvo un período de existencia de muchos miles de años, mientras que la "Química" oficialmente establecida como una ciencia en el siglo XVIII sólo de unos trescientos, hecho que pone de manifiesto la importancia de la tan a menudo poco reconocida Al-Chymia.

Aún así, la tarea de conseguir aislar los elementos químicos ha sido muy ardua y ha necesitado una gran cantidad de esfuerzos durante mucho tiempo. En 1250 solamente se conocían 10 elementos y hubo que esperar hasta 1669 para identificar 14. En 1771 se llegó a los 20, y a 62 en 1868. En 1935, en pleno desarrollo de la mecánica cuántica ondulatoria, se alcanzaban los 88 , y se llegó a los 109 en 2006 (Scerri, 2011). En 2014 ya se han podido obtener muestras de los 118 elementos que conforman la Tabla Periódica, aunque algunos de ellos hayan tenido una vida extremadamente corta (Winter, 2014).

Si realizamos el balance de la progresión del descubrimiento y aislamiento de los elementos químicos a lo largo de la Historia observaremos los resultados que se muestran en la figura 7. En ella se puede comprobar el elevado número de elementos obtenidos en los siglos XIX y XX, reflejo del perfeccionamiento de las técnicas instrumentales que facilitaron esta labor.

\section{AGRADECIMIENTOS}

La autora desea agradecer a los referees anónimos de la revista por sus acertadas sugerencias, que mejoraron el manuscrito original y lo dejaron listo para su publicación.

\section{Referencias}

Al-Khalili, J. (2012). The house of wisdom: how Arabic science saved ancient knowledge and gave us the Renaissance. Penguin Books, New York. 336 pp.

Bensinger, H. (2014). Ancient Greek technology. PowerKids Press, New York, 24 pp.

Cannizzaro, E. (2009) Compendio de un curso de filosofía química. Traducido, comentado y editado por P. Román, Prensas Universitarias de Zaragoza, Zaragoza, 139 pp.

Castillo Martos, M. (2005). Creadores de la ciencia moderna en España y América: Ulloa, los Delhuyar y del Río descubren el platino, el wolframio y el vanadio. Muñoz Moya Editores Extremeños, Badajoz, 293 pp.

Castillo Martos, M. (2006). Bartolomé de Medina y el Siglo XVI. Universidad de Cantabria, Santander, 303 pp.

Chakravarti, B. (2003). Mātrkā-bheda Tantram: A Book on the Ancient Indian Chemistry. Self Employment Bureau Publication, Kolkata, 103 pp.

Emsley, J. (2000). La Impactante Historia del Fósforo: Una Biografía del Elemento del Diablo. Macmillan, Londres, $336 \mathrm{pp}$.

Ercker, L. (1736). Aula subterranea. Das ist: Untererdische Hofhaltung. Oder Gründliche Beschreibung dererjenigen Sachen, so in der Tieffe der Erden wachsen, als aller Ertzen der Königlichen und gemeinen Metallen, auch fürnehmster Mineralien. Nach möglichstem Fleiß und Sorgfalt vermehrt und verbessert durch J.E.C. Jung J.D., Frankfurt, 245 pp.

Fernández Castro, M.C. (1997). La Prehistoria de la Península Ibérica. Editorial Crítica, Barcelona, 396 pp.

Gago, R. \& Pellón, I. (1994). Historia de las cátedras de Química y Mineralogía de Bergara a finales del siglo XVIII. Ayuntamiento de Bergara, Bergara, 201 pp.

Ghiorso, A., et al. (1955). New Elements Einsteinium and Fermium, Atomic Numbers 99 and 100. Physical Review, 99(3): 1048-1049. http://dx.doi.org/ 10.1103/PhysRev.99.1048.

Guyton De Morveau, L.B. (1787) Methode Nomenclature Chimique. Chez Cuchet, Paris, 314 pp.

Greenberg, A. (2007). From alchemy to chemistry in picture and story. Wiley-Interscience, Hoboken, New Jersey, 600 pp.

Heines, V. (1958). Libellus de alchimia: ascribed to Albertus Magnus. Translated from the Borgnet Latin Edition, Introduction and notes by Sister Virginia 
Heines, etc. University of California Press, Berkeley \& Los Angeles, 79 pp.

IUPAC (2005). Nomenclature of Inorganic Chemistry IUPAC. Recommendations 2005. The Royal Society of Chemistry, London, 366 pp.

Kirk, R.E.; Othmer, D.F. \& Seidel, A. (2007). KirkOthmer Encyclopedia of Chemical Technology, Wiley-Interscience, New Jersey. 5th ed., 22950 pp.

Klemm, R. \& Klemm, D. (2013). Gold and Gold Mining in Ancient Egypt and Nubia: Geoarchaeology of the Ancient Gold Mining Sites in the Egyptian and Sudanese Eastern Deserts. Springer-Verlag, Berlín, 649 pp. http://dx.doi.org/10.1007/978-3-642-22508-6.

Lavoisier, A. (1789), Traité Élémentaire de Chimie, présenté dans un ordre nouveau, et d'après des découvertes modernes. Cuchet, Paris, 331 pp.

Lémery, N. (1703). Curso Chymico. Duveen, 348 pp.

López Piñero, J. M. (1979). Ciencia y técnica en la sociedad española de los siglos XVI y XVII. Labor, Barcelona, $511 \mathrm{pp}$.

Martín Reyes, G. (2004). Breve historia de la Alquimia. Fundación Canaria Orotava de Historia de la Ciencia, La Orotava, 108 pp.

Martinón-Torres, M., et al. (2003). A 16th century lab in a 21 st century lab: archaeometric study of the laboratory equipment from Oberstockstall (Kirchberg am Wagram, Austria). Antiquity, 77(298), http://bit. ly/1bwLzc4.

Martinón-Torres, M. (2008). Los orígenes alquímicos de la química moderna: una perspectiva arqueológica. Anales de Química, 104(4): 310-317.

McDonald, D. \& Hunt, L. B. (1982). A History of Platinum and its Allied Metals. Johnson Matthey, London, $450 \mathrm{pp}$.

Moorey, P.R.S. (1994). Ancient Mesopotamian Materials and Industries: the Archaeological Evidence. Clarendon Press, New York, 415 pp.

Morss, L.R.; Edelstein, N.M. \& Fuger, J. (Eds). (2011) The Chemistry of the Actinide and Transactinide Elements. Springer Netherland, Dordrecht, 4514 pp.

Needham, J. (1978). De la ciencia y la tecnología chinas. Siglo XXI, México, $4^{\mathrm{a}}$ ed., 243 pp.

Newman, W.R. \& Principe, L.M. (1998). Alchemy vs. Chemistry: The Etymological Origins of a Historiographic
Mistake. Early Science and Medicine, 3: 32-65. http://dx.doi.org/10.1163/157338298X00022.

Pellón González, I. (2012). El atomismo en química. Un Nuevo Sistema de Filosofía Química de John Dalton. Universidad de Alicante, Alicante, $163 \mathrm{pp}$.

Pellón González, I. (2013). De wolframio a tungsteno: Reflexiones en el 230 aniversario de su aislamiento por Juan José y Fausto Delhuyar. Tierra y Tecnología, 43: 41-53.

Pellón González, I. \& Bilbao-Goyoaga, A. Ma (2013). The chemical atomic theory in Ramón Torres Muñoz de Luna's textbooks (1848-1885). Circumscribere: International Journal for the History of Science, 13: 46-65.

Pérez Pariente, J. \& López Pérez, J. (2005). Alquimia: ciencia y pensamiento a través de los libros. Catálogo de la exposición celebrada en la Biblioteca Histórica "Marqués de Valdecilla". Servicio de Publicaciones de la Universidad Complutense de Madrid, Madrid, $214 \mathrm{pp}$.

Pérez Pariente, J. (2010). La alquimia y su relación con la ciencia. Ábaco, 63: 23-31.

Priesner, C. \& Figala, K. (1998). Alchemie, Lexikon einer hermetischen Wissenschaft. C.H. Beck, Munich, $412 \mathrm{pp}$.

Príncipe, L.M. (2013). The Secrets of Alchemy. The University of Chicago Press, Chicago and London, $281 \mathrm{pp}$.

Román, P. (2008). Mendeléiev. El profeta del orden químico. Nívola, Madrid, $224 \mathrm{pp}$.

Scerri, E. (2011). The Periodic Table: A Very Short Introduction. Oxford University Press, Oxford, $168 \mathrm{pp}$. http://dx.doi.org/10.1093/actrade/9780199582495. 001.0001 .

Thierfelder, C. (2008). Dirac-Hartree-Fock studies of $\mathrm{X}$-ray transitions in meitnerium. The European Physical Journal, 36(2): 227-231. http://dx.doi.org/10. 1140/epja/i2008-10584-7.

UAB (2011). El laboratori d'Andreas Libavius. http:// www.bib.uab.cat/ciencies/expo/quimica/hq2.htm.

Weeks, M. E. (1932). The discovery of the elements. VIII. The platinum metals. Journal of Chemical Education, 9 (6): 1017-1034. http://dx.doi.org/10.1021/ed009p1017.

Winter, M. (2014). WebElements: the periodic table on the web. http://www.webelements.com. 